\title{
Role of WAN Optimization Solutions in overcoming limitations of TCP performance in Ad-Hoc Networks
}

\author{
ANIKET DESHPANDE \\ Dept. of CSE, Mewar University, Rajasthan, India \\ E-mail: anik.deshpande@gmail.com
}

\begin{abstract}
Ad-hoc network is a temporary network that is designed for a special purpose and is developed as soon as the various devices are connected to each other. This type of network degrades the performance of TCP or transmission control protocol which is the core element of internet protocol. The performance of TCP can be enhanced by using various optimization techniques. This paper focuses on the feasibility, advantages and disadvantages of making use of contemporary WAN optimization technologies to overcome the challenges of degrading the performance of TCP in Ad-Hoc Networks. Moreover, the paper will also highlight the significance of the use of Ad-hoc networks by end users in accessing various cloud based services.
\end{abstract}

Keywords: Ad-Hoc Network, Transmission Control Protocol, WAN optimization, \& Cloud based services.

\section{INTRODUCTION}

Ad-hoc network refers to the local area network which is developed as soon as the various devices are connected to each other. This type of network does not rely on a base station; instead data packets are sent and received by individual network nodes [1]. However, this type of network affects the performance of TCP or transmission control protocol which is the core element for maintain communication in a network. Basically, TCP manages the transmission of data packets between the computers and allows the exchange of data between two hosts. It also assures that data is received in the same pattern in which it is sent [1, 2]. Transmission control protocol is an essential part of the internet protocol, hence it is very important to monitor its performance.

However, the performance of Transmission Control Protocol is affected while using ad-hoc network. This happens because of various problems associated with the ad-hoc networks. One of the major problems is that the medium of radio is unforeseeable [3]. Moreover, many other issues such as node motilities and jamming of the network also affect the performance of TCP and also it appears to be a very tedious task to address and solve these issues. In addition, the performance of TCP is also affected quite negatively because of links break down that occur recurrently [3, 4]. As a consequence, it becomes very difficult for the TCP to successfully transfer data packets through its transmitters. Thus, the overall efficiency of transmission control protocol declines significantly.

The performance of TCP can be enhanced remarkably while using ad-hoc network through various optimizations [2]. One such optimization is WAN Optimization which helps in overcoming the limitations of TCP performance in ad-hoc Networks. WAN optimization solutions increase the overall efficiency of TCP with ad-hoc networks and it controls and manages the data traffic in such a way that all the data is delivered to the users quite swiftly [5]. It also ensures that the delivery of data to the users is secure and safe.

\section{TCP PERFORMANCE IN AD-HOC NETWORKS}

Ad-hoc networks are composed of static nodes and hence are complex distributed systems. The presence of static nodes allows this type of network to be dynamic and therefore it can organize itself freely. One more distinguishing feature of Ad-hoc networks is that there is no availability of base station facility which can support any type of communication $[6,7]$. Moreover, the ways in which 
the various components of the network are integrated together also differ on the type of environment. Due to all of these features of the Adhoc network, the performance of the transmission control protocol also differs from its usual performance in this type of network [4]. To be more precise, the performance of TCP declines in this type of network. Lossy channels, nonsymmetric paths, hidden and exposed stations, power constraints and route failures are some of the major issues that TCP faces in Ad-hoc networks.

Furthermore, it becomes very tedious to deal with these problems when TCP is used with Ad-hoc networks. Although there are various methods and protocols that can be used to cop up with these problems, not all protocols yield fruitful results as each of the methods carries several other disadvantages with them [3]. Thus it becomes extremely important to pay due consideration to the selection of the best protocol in order to remove the limitations of the usage of TCP with Ad-hoc networks. Ad-hoc networks are basically temporary networks that are designed for a special purpose and do not require any previous framework. So, if a proper technique is used for making TCP compatible with Ad-hoc networks, these networks can be used more commercially [7]. Because of the significance of Ad-hoc networks, various researches have been conducted to analyze the feasibility of TCP with Ad-hoc networks.

The basic objective behind the development of transmission control protocol or TCP is to ensure that all the users can receive data with sufficient reliability. However, the implementation of the TCP requires extensive require $[1,8]$. This is because of the fact that when TCP was designed, it was the foremost assumption that this protocol would serve the wireless networks. If this assumption is neglected during the implementation of TCP, it can lead to erroneous performance and results [8]. Therefore, whenever TCP is used with Ad-hoc networks, the major problem is the obstruction control which arises because of the losses that are not prompted by the network itself. Moreover, all the TCPs that are used today are based on the basic assumption that these losses occur because of the obstruction [9]. It is due to the fact that in any type of network that is based on wired networking approach, the rates of bit error are very low.

In addition, the losses that cause the obstruction control arise because of the loss of various data packets. These data packets are sometimes lost by the time out approach and sometimes by the duplicated approach [3,4]. As a consequence, the sending rate is decreased significantly by TCP. This slowing down is caused by the variations and adjustments in the congestion or obstruction window. Since, the TCP is designed on the assumption of wired networking approach; it becomes very difficult for TCP to perform efficiently in an Ad-hoc networking environment [6]. In order to overcome the problems associated with the usage of TCP in Ad-hoc networks, it requires various other technologies to be optimized for sound and efficient performance of transmission control protocol. The use of optimized technologies allows controlling the losses of data packets that arise during the use of TCP in Ad-hoc networks. WAN optimization techniques are proving to very helpful in overcoming these problems without any significant decrease in the overall performance of transmission control protocol in Ad-hoc networks $[3]$.

\subsection{Ad Hoc Networks and Challenges of TCP}

It has been observed that the performance of TCP declines significantly in ad-hoc networking environment. This happens because of a number of issues that are associated with ad-hoc networks [4]. Among various problems that affect the performance of TCP, the major problems are discussed in the below section.

\subsubsection{Lossy Channels}

The main issue associated with the use of TCP in Ad-hoc networks is the occurrence of lossy channels. The major reason behind these lossy channels is the attenuation of signals [9]. This means that the intensity of the electromagnetic waves decreases at the receiver which ultimately leads small ratios of signal to noise. Another reason behind the lossy channels is due to a very well known effect known as the Doppler Effect. This effect refers to the variation in the relative velocities of the receiver and the transmitter [1]. Because of Doppler Effect, the frequencies of the signals vary or shift, hence the recording of signals becomes very difficult. Moreover, multipath fading also contributes to the occurrence of lossy channels. In this process, the path of the electromagnetic waves deviates and changes due to reflection and diffraction. This can cause the waves to reach the receiver through numerous paths, hence resulting in variations in amplitude and other important properties of the signals (8). All these processes lead to lossy channels which can severely degrade the performance of TCP in Ad-hoc networks. 


\subsubsection{Hidden and Exposed stations}

Another problem faced by TCP in Ad-hoc networks is the presence of hidden and exposed channels [7]. It is due to the fact that Ad-hoc networks rely on various sensing mechanisms in order to detect the best channel for communication. However, one of the major problems associated with the use of these mechanisms is that it is not possible to adequately solve the issues of hidden and exposed stations [6]. Thus, the range of transmission of data packets cannot be determined accurately with these mechanisms.

\subsubsection{Path asymmetry}

TCP also faces the challenge of non uniformity of paths in Ad-hoc networking environment. The non uniformity of paths may occur in various forms such as non uniformity of bandwidths, non uniformity of loss rate and non uniformity of routes [3]. In case of non uniformity of bandwidths, the networks using satellites face problems in locating a symmetric bandwidth. This problem arises because of various technical factors such as mass, volume, etc. Hence, the use of return links is completely omitted which causes the problem to occur. Another form of non uniformity in Ad-hoc networks is the non uniformity of transmission and loss rates [4]. This factor ultimately leads to the bandwidth non uniformity in Ad-hoc networks. All of the above mentioned non uniformities contribute significantly to degrading the performance of TCP in Ad-hoc networks.

\subsubsection{Loss Rate Asymmetry}

Among various other non uniformities mentioned earlier, the non uniformity of loss rates also affects the performance of TCP in Ad-hoc networks [1]. The major reason behind the non uniformity of loss rates is that the backward path on some occasions becomes more lossy as compared to the forward path. This problem arises because of the fact that Ad-hoc networks are designed on the principle of dependency of pocket losses on regional limitations [6]. It means that the packet losses differ at different locations. In some cases, the non uniformity of loss rates can also lead to the occurrence of non uniformity of bandwidths which further degrades the performance of TCP in Ad-hoc networks.

\subsubsection{Route asymmetry}

The non uniformity of routes also degrades the performance of TCP in Ad-hoc networks. In this category of non uniformity, the forward and the backward paths differ from each other. It means that the routes of TCP data and its acknowledgements vary from each other. This type of non uniformity usually arises because of the design of the protocol that is used for the purpose of routing [4, 9]. Moreover, the situation becomes more adverse when losses of data pockets increases in case of movement from one location to another. The loss of data pockets occur because movement from one location to another results in the variation of backward and forward routes, thus, degrading the overall performance of TCP in Ad-hoc networks [9]. Nevertheless, in case of static networks where mobility is almost negligible, the loss of data pockets is also very low. Therefore, extensive care is required in selecting the Ad-hoc networks with high mobility in order to maintain performance of TCP.

\subsubsection{Power constraints}

Another challenge that is faced by transmission control protocol in Ad-hoc networks is the constraints and limitations associated with the power capabilities of batteries in mobile devices with Ad-hoc networks. This problem is very critical in determining the performance of TCP as every node in the network has to perform two functions [7]. First of all, each node has to act as an end system and secondly, it has to serve the purpose of router too. Thus, additional power requirements arise in sending the data packets in both backward and forward direction. This makes the available power very scarce $[6,1]$. So, transmission control protocol must use this scarce power in such a way that it does not affect the overall efficiency of the protocol.

The availability of scarce power in Ad-hoc networks is a very big challenge for TCP. Moreover, in Ad-hoc networks, the major problems associated with power are power saving and power control [5]. Power saving refers to use minimum power in the overall operations, whereas, power control means to ensure that all the nodes in the network are being provided with adequate amount of power [4, 8]. Therefore, it appears quite necessary to properly address these power problems in Ad-hoc networks in order to avoid any sort of degradation in the performance of TCP.

\section{WAN OPTIMIZATION}

WAN optimization refers to the adoption of techniques and methods which can enhance the efficiencies related to the transfer of data over a wide area network. In today's digital world, the need of WAN optimization is growing quite rapidly 
due to its large applications in various domains of technology. One of the greatest significance of WAN optimization is that it can increase the overall efficiency of TCP in Ad-hoc networks and remove various limitations that TCP has to deal with in an Ad-hoc networking environment [2, 5]. WAN optimization can enhance the performance of TCP is a number of ways such as fulfilling the requirements of bandwidths, optimizing the latency, dealing appropriately with bottlenecks, etc. Various studies have been conducted and are still in progress on the subject of WAN optimization and its significance [2]. The ultimate target of WAN optimization is to benefit both the commercial and public sectors.

WAN optimization helps in enhancing the endto-end throughput of data in networks with transmission control protocol. However, the type of wide area network defines the optimization solutions to be implemented. The first type of WAN is branch to headquarter and the other one is Data Center to Data Center which is more commonly known as DC2DC WAN. In the first type of WAN there are small live connections, less bandwidths and various protocols available [2]. This type of WAN is most used for commercial and business purposes. On the other hand, in the later type of WAN, there are more bandwidths, large connections, and data migration and backup facility. TCP performance can be enhanced by optimizing any of the one type of WAN depending on the availability and compatibility. In the past, techniques and solutions were present for the optimization of branch to headquarter type of WAN [5]. However, in the recent years, techniques have been developed for optimizing the DC-2-DC type of WAN as well.

\subsection{Role of WAN Optimization Solution to address the Performance Bottlenecks}

The performance of transmission control protocol is affected quite negatively in an Ad-hoc networking environment. So it appears to be quite essential to maintain and enhance the efficiency of TCP in Ad-hoc networks. The limitations of TCP in Ad-hoc networks can be removed best by the implementation of WAN optimization solutions [8]. In today's digital world, nom organization can survive and compete without WAN optimization. Thus it has become the focus of every organization to implement the WAN optimization solutions in order to overcome the limitations of TCP in Ad-hoc networks $[9,10]$. WAN optimization solutions are basically integrated applications of networking strategies which can significantly increase the transfer of data over the network [4]. The performance of TCP can be enhanced in Ad-hoc networks by the implementation of various techniques and solutions which have been discussed below.

TCP performance can be improved significantly by the implementation of deduplication technique. It is a method of WAN optimization in which the transfer of unnecessary data across the network is completely eradicated. In this way, all the relevant data is saved from being ignored and overall efficiency of TCP also increases [9]. Another important technique is the compression method. This technique refers to compressing and minimizing the size of the data files that are transferred across the network. It helps to accelerate the rate of data transfer and saving the precious time that would have been otherwise consumed. Moreover, the performance of TCP can also be increased remarkably by the optimizing the latency [4]. This technique involves the filtration of TCP which can be achieved by scaling of the window size, picking up appropriate acknowledgements, controlling the obstructions or congestions, re locating the application in the vicinity of the end user, etc. All these techniques assist in optimizing the latency [10]. In some cases, the latency can also be optimized by the implementation of various mechanisms which reduce the WAN latency.

Furthermore, caching is also a good technique of optimization. It involves approaching the same data again and again [8]. In addition, the technique of correcting errors is also a useful tool. It helps in providing the substitute data packets for the data packets that have been lost during the transmission over the network. More so, controlling the traffic of data also increases the overall efficiency. It involves controlling each type of data for every different application $[8,10]$. It helps in avoiding any application from dominating any other application in the network. This type of WAN technique can be attained by the use of various devices available for accelerating WAN [10]. Another important strategy can be equalizing and setting priorities for every individual type of data. Moreover, limiting the connections is also an important tool for optimizing WAN which ultimately enhances the performance of TCP in Adhoc networks. Likewise, predefining and setting simple rate limits can also enhance the performance of TCP in Ad-hoc networks [4]. This can be achieved by allowing only a fix amount of data to be delivered to any individual user in the network. 


\subsection{Pros and Cons of Contemporary WAN Optimization Technologies}

Current WAN optimization technologies have numerous advantages as compared to the disadvantages. First of all, these technologies enable much faster accessibility to the data files [2]. This is possible because of the use of caching technique which allows the same files to be accessed again without reloading and hence significant amount of time is saved. Secondly, these technologies have eliminated the limitation of location of the users [5]. Any user can transfer and access the data at very high speeds irrespective of locations. Moreover, allocation of bandwidths to more critical applications has become possible which enhances the overall performance. In addition, these technologies have also enabled backup and recovery of data files much easier and convenient. Likewise, latency optimization allows the maximum use of bandwidths which increases the overall efficiency [5]. These technologies reduce the costs associated with bandwidths and software, thus the overall costs also decrease significantly.

One of the major disadvantages of implementing WAN optimization techniques is that monitoring of both the ends becomes necessary. Every individual site needs an optimizing technique which can increase the costs. In addition, management becomes more complicated because of more hardware involved $[2,5]$. Moreover, variations in processing power and throughput can also require a lot of machines to be installed on an individual host. Therefore, the ultimate objective of increasing the overall efficiency might not be achieved because of the presence of numerous machines on a single host.

\subsection{Significance of AD-Hoc Networks by End Users in accessing Cloud based Services}

In today's digital world, cloud based services have revolutionized the methods of sharing computing resources. This technology has eliminated the need to handle various applications on smart devices, thus making the mobile and smart devices more reliable [11]. The computing features of smart devices have enabled the creation of networks based on cloud technology. However, sometimes it is not possible for smart devices to create such networks and organize them [12]. This limitation can be overcome by the use of Ad-hoc networks in accessing various cloud based services such as Office 365, Amazon cloud, etc. The ad-hoc network can be created spontaneously at any time in order to access these services.
Ad-hoc network enables the utilization of cloud technology in such a way that helps to make full use of various resources that are untapped. Moreover, these resources can be utilized from the hardware that is usually not dedicated $[11,12]$. The use of Ad-hoc networks in accessing cloud based services can enable various organizations to reduce the overall costs of cloud computing technology. Furthermore, a large number of cloud based services can be accessed by the end users with the use of Ad-hoc networks as compared to the conventional cloud network with no Ad-hoc network [11]. In addition, the use of Ad-hoc networks in cloud technology can also reduce the overall expenditure of energy.

\section{CONCLUSION}

It can be concluded from the above discussion that TCP performance degrades in Ad-hoc networks due to several reasons including lossy channels, path asymmetry, hidden and exposed stations, network partitions, power constraints and route failures. Nevertheless, WAN optimization solutions assist in overcoming the limitations of TCP performance in Ad-hoc networks. Besides, Ad-hoc networks are also beneficial for end users in accessing various cloud based services.

\section{REFERENCES}

[1] Perkins CE. Ad Hoc Networking. AddisonWesley Professional; 2008 Aug 7 [cited 2014 Nov 14]; Available from: http://dl.acm.org/citation.cfm?id=1481270

[2] Jr. TG, 15311 JCCN. Application Acceleration and WAN Optimization Fundamentals [Internet]. Cisco Press; 2012 [cited 2014 Nov 14]. Available from: http://books.google.com/books?hl=en\&lr=\&id $=\mathrm{eSkbCy} 4 \mathrm{wEtMC} \&$ pgis $=1$

[3] Ilyas M (Ed. . The Handbook of Ad Hoc Wireless Networks [Internet]. CRC Press; 2014 [cited 2014 Nov 14]. Available from: http://books.google.com/books?hl=en\&lr=\&id $=710$ sWB-q6EMC \&pgis $=1$

[4] Fall KR, Stevens WR. TCP/IP Illustrated, Volume 1: The Protocols [Internet]. AddisonWesley; 2011 [cited 2014 Nov 14]. Available from:

http://books.google.com/books?hl=en\&lr=\&id $=\mathrm{a} 23 \mathrm{OAn} 5 \mathrm{i} 8 \mathrm{R} 0 \mathrm{C} \&$ pgis $=1$

[5] Zhang Y, Ansari N, Wu M, Yu H. On Wide Area Network Optimization. IEEE Commun Surv Tutorials [Internet]. IEEE; 2012 [cited 2014 Nov 14];14(4):1090-113. Available 
A. Deshpande / International Journal of Computer Networks and Communications Security, 2 (11), November 2014

from:

http://ieeexplore.ieee.org/articleDetails.jsp?arn umber $=6042388$

[6] Leung K, Li V, Yang D. An Overview of Packet Reordering in Transmission Control Protocol (TCP): Problems, Solutions, and Challenges. IEEE Trans Parallel Distrib Syst [Internet]. IEEE; 2007 Apr 1 [cited 2014 Nov 14];18(4):522-35. Available from: http://ieeexplore.ieee.org/articleDetails.jsp?arn umber $=4118693$

[7] Christian Lochert BSMM. A Survey on Congestion Control for Mobile Ad-Hoc Networks. [cited 2014 Nov 14]; Available from: http://citeseerx.ist.psu.edu/viewdoc/summary?d oi $=10.1 .1 .72 .7225$

[8] Singh R. Improving TCP Performance in Multi Hop Mobile Adhoc Network. Int J Electron Comput Sci Eng [Internet]. 2012 [cited 2014 Nov 14]; Available from: http://www.ijecse.org/wpcontent/uploads/2012/01/PP-109-114.pdf

[9] Rahman A, Islam S, Talevski A. Performance measurement of various routing protocols in ad-hoc networks. 2009 [cited 2014 Nov 14]; Available from: http://espace.library.curtin.edu.au/R?func=dbin -jump-full\&local_base $=$ gen 01 era02\&object_id $=132629$

[10]Langer SG, French T, Segovis C. TCP/IP optimization over wide area networks: implications for teleradiology. J Digit Imaging [Internet]. 2011 Apr [cited 2014 Nov 14];24(2):314-21. Available from: http://www.pubmedcentral.nih.gov/articlerende r.fcgi?artid $=3056981 \&$ tool $=$ pmcentrez\&render type $=$ abstract

[11]Berl A, Gelenbe E, Di Girolamo M, Giuliani G, De Meer H, Dang MQ, et al. Energyefficient cloud computing. Comput J. 2010;53:1045-51.

[12] Aymerich FM, Fenu G, Surcis S. An approach to a cloud computing network. 1st International Conference on the Applications of Digital Information and Web Technologies, ICADIWT 2008. 2008. p. 113-8. 